\title{
Multi-Step Iteration Algorithm of Total Asymptotically Quasi-Nonexpansive Maps
}

\author{
Salwa Salman Abed*, Zahra Mahmood Mohamed Hasan
}

Department of Mathematics, college of Education for Pure Sciences (Ibn Al-Haitham) / University of Baghdad, Baghdad, Iraq

\begin{tabular}{l} 
A R T I C L E I N F O \\
\hline Article history: \\
Received: 14 February 2019 \\
Accepted: 28 April, 2019 \\
Online: 21 May, 2019 \\
\hline Keywords: \\
Banach space, \\
total asymptotically quasi- \\
nonexpansive map, \\
weak convergence, \\
strong convergence, \\
common fixed points \\
\hline
\end{tabular}

\section{Introduction and Preliminaries}

This paper was originally published in the Conference: 2018 International Conference on Advanced Science and Engineering (ICOASE), Iraq [1]. It is well known that the concept of asymptotically nonexpansive introduced by Goebel and Kirk [2]. Additionally, every asymptotically nonexpansive map of a Banach space has a fixed point is proved. In [3], Petryshyn and Williamson proved the weak and strong convergence for quasi-nonexpansive map by using a sufficient and necessary condition. Alber [4], a new class of asymptotically nonexpansive is introduced. As well as, approximating methods for finding their fixed points are studied. In 2014, G. S. Saluja [5] established the strong and weak convergence for approximating common fixed point for generalized asymptotically quasi-nonexpansive maps in a Banach space.

Very recently, In [6], the authors proposed an implicit iteration for two finite families of generalized asymptotically quasinonexpansive maps. As well as, some strong convergence theorems are established. It is useful to point out our findings in this area which appeared in [7].

Let $\mathrm{B}$ be a non-empty closed convex subset of a real Banach space $M$ and $T$ be a self-map of $B$. The set of all fixed points denoted by $F(T)$. A self-map $T$ from $B$ into $M$ is called nonexpansive map [2] if

\footnotetext{
*Salwa Salman Abed, Email: salwaalbundi@yahoo.com
}

$$
\|T a-T b\| \leq\|a-b\| \text { for all } a, b \in B
$$

and is called quasi- nonexpansive map [6] if $F(T) \neq \varnothing$ and

$$
\left\|T a-a^{*}\right\| \leq\left\|a-a^{*}\right\|
$$

for all $a \in B$ and for all $a^{*} \in F(T)$.

A Banach space $M$ is satisfying:

- "Opial's condition if for each sequence $\left(a_{n}\right)$ in $M$, is weak convergence to $a$ implies that

$$
\lim _{n \rightarrow \infty} \inf \left\|a_{n}-a\right\|<\lim _{n \rightarrow \infty} \inf \left\|a_{n}-b\right\|
$$

for all $b \in M$ with $a \neq b "$.

- $\quad$ "Kadec-Klee property if for each sequence $\left(a_{n}\right)$ in $M$ is weak convergence to $(a)$ together with $\left\|a_{n}\right\|$ converges strongly to $\|a\|$ imply that $\left(a_{n}\right)$ is strong convergence to a point $a \in M[7] "$.

The aim of this paper, an iterative scheme for two families of total asymptotically quasi-nonexpansive maps is established. The strong and weak convergence theorems of this scheme for approximation of common fixed points in Banach space by using suitable conditions are established. For this purpose, let us recall the following definitions and lemmas.

Definition (1.1): "A map $T$ is named asymptotically nonexpansive [1] if there is a sequence $\left(f_{n}\right)$ in $[0,+\infty)$ with $\lim _{n \rightarrow \infty} f_{n}=0$ and $\left\|T^{n} a-T^{n} b\right\| \leq(1+$ $\left.f_{n}\right)\|a-b\|$, for all $a, b \in B, n=1,2, \ldots$ 
If $F(T) \neq \varnothing$ there is a sequence $\left(f_{n}\right)$ in $[0,+\infty)$ with $\lim _{n \rightarrow \infty} f_{n}=$ 0 and $\left\|\mathrm{T}^{\mathrm{n}} \mathrm{a}-\mathrm{a}^{*}\right\| \leq\left(1+\mathrm{f}_{\mathrm{n}}\right)\left\|\mathrm{a}-\mathrm{a}^{*}\right\|$,

for all $\mathrm{a} \in \mathrm{B}, \mathrm{a}^{*} \in \mathrm{F}(\mathrm{T})$ and $\mathrm{n}=1,2, \ldots$. Therefore, $\mathrm{T}$ is named asymptotically quasi-nonexpansive map [10]".

Definition (1.2): "A map T is named total asymptotically nonexpansive map [4] if there are null sequences of postive real numbers $\left(\mathrm{f}_{\mathrm{n}}\right)_{\mathrm{n}=1}^{\infty},\left(\mathrm{g}_{\mathrm{n}}\right)_{\mathrm{n}=1}^{\infty}, \mathrm{n} \geq 1$ and nondecreasing continuous function $\psi:[0, \infty) \rightarrow[0, \infty)$ with $\psi(0)=0$ such as $\forall a, b \in B$

$$
\left\|\mathrm{T}^{\mathrm{n}} \mathrm{a}-\mathrm{T}^{\mathrm{n}} \mathrm{b}\right\| \leq\|\mathrm{a}-\mathrm{b}\|+\mathrm{f}_{\mathrm{n}} \psi\|\mathrm{a}-\mathrm{b}\|+\mathrm{g}_{\mathrm{n}}{ }^{\prime} .
$$

$\mathrm{T}$ is named total asymptotically quasi-nonexpansive map if $\mathrm{F}(\mathrm{T}) \neq \emptyset$, there are null sequences of postive real numbers $\left(f_{n}\right)_{n=1}^{\infty},\left(g_{n}\right)_{n=1}^{\infty}, n \geq 1, \sum_{n=1}^{\infty} f_{i n}<\infty$ and $\sum_{n=1}^{\infty} g_{\text {in }}<\infty$, and nondecreasing continuous function $\psi:[0, \infty) \rightarrow[0, \infty)$ with $\psi(0)=0$ such as $\forall a \in B$ and $a^{*} \in F(T)$

$\left\|\mathrm{T}^{\mathrm{n}} \mathrm{a}-\mathrm{a}^{*}\right\| \leq\left\|\mathrm{a}-\mathrm{a}^{*}\right\|+\mathrm{f}_{\mathrm{n}} \psi\left\|\mathrm{a}-\mathrm{a}^{*}\right\|+\mathrm{g}_{\mathrm{n}}$.

If $g_{n}=0, \forall n=1,2, \ldots$ therefore $\mathrm{T}$ is asymptotically quasinonxpansive map.

Definition (1.3)[11]: "Let $B$ be a nonempty closed convex subset of a Banach space $M$. A self-map $T$ is named uniformily $K$-Lipschitzain if there exists a constant $K>0$ such that $\left\|T^{n} a-T^{n} b\right\| \leq K\|a-b\|, \forall a, b \in B$.

Definition (1.4)[12]: "A map $T: B \rightarrow M$ is named demi-closed with respect to $b \in M$ if for each sequence $\left(a_{n}\right)$ in $B,\left(a_{n}\right)$ is weak convergence to $a$ and $T\left(a_{n}\right)$ is strong convergence to $b$. Hence $a \in B$ and $T(a)=b$. If $(I-T)$ is demiclosed which means if $\left(a_{n}\right)$ is weak convergence to $a$ in $B$ and $(I-T)$ is strong convergence to 0 . Therefore $(I-T)(a)=0 "$.

Note: Now to explain the relation between the above definitions:

$\begin{array}{cc}\text { Nonexpansive } \Rightarrow \begin{array}{c}\text { Asymptotically } \\ \text { nonexpansive }\end{array} & \begin{array}{c}\text { Total asymptotically } \\ \text { nonexpansive }\end{array} \\ \sqrt{\Omega} & \sqrt{\Omega} \\ \text { Quasi- } \Rightarrow \text { Asymptotically quasi } & \text { Total asymptotically } \\ \text { nonexpansive -nonexpansive } & \text { quasi - nonexpansive }\end{array}$

Lemma (1.5)[13]:" Let $M$ be a uniformly convex Banach space and $0<L \leq t_{n} \leq K<1, \forall n \in N$. Presume that $\left(a_{n}\right)$ and $\left(b_{n}\right)$ are two sequences of $\mathrm{M}$ such as:

$\lim _{n \rightarrow \infty}\left\|a_{n}\right\| \leq r, \lim _{n \rightarrow \infty}\left\|b_{n}\right\| \leq r$ and $\lim _{n \rightarrow \infty}\left\|t_{n} a_{n}+\left(1-t_{n}\right) b_{n}\right\|=$ $r$

hold for some $r \geq 0$. Thus $\lim _{n \rightarrow \infty}\left\|a_{n}-b_{n}\right\|=0 "$.

Lemma (1.6)[14]: "Let $\left(\mu_{n}\right)_{n=1}^{\infty},\left(\sigma_{n}\right)_{n=1}^{\infty}$ and $\left(e_{n}\right)_{n=1}^{\infty}$ be sequences of postive numbers accomplishing the following inequality:

$$
\mu_{\mathrm{n}+1} \leq\left(1+\sigma_{\mathrm{n}}\right) \mu_{\mathrm{n}}+\mathrm{e}_{\mathrm{n}}, \forall \mathrm{n} \geq 1
$$

if $\sum_{\mathrm{n}=1}^{\infty} \sigma_{\mathrm{n}}<\infty$ and $\sum_{\mathrm{n}=1}^{\infty} \mathrm{e}_{\mathrm{n}}<\infty$, then

$\left(\mu_{\mathrm{n}}\right)$ is bounded and $\lim \mu_{\mathrm{n}}$ exists. In additional

if, $\lim _{n \rightarrow \infty} \inf \mu_{n}=0$ then $\lim _{n \rightarrow \infty} \mu_{n}=0$ ".

Lemma (1.7)[15]: "Let $B$ be a nonempty convex subset of a uniformly convex Banach space. Therefore there exists a strictly nondecreasing continuous function $f:[0, \infty) \rightarrow[0, \infty)$ with $f(0)=0$ such as for each Lipschitzain map $T: B \rightarrow B$ with Lipschitz constant K:

$$
\begin{aligned}
& \| \mathrm{tTa}+(1-\mathrm{t}) \mathrm{Tb}-\mathrm{T}(\mathrm{ta}+(1-\mathrm{t}) \mathrm{b} \| \\
& \leq \mathrm{Kf}^{-1}\left(\|\mathrm{a}-\mathrm{b}\|-\frac{1}{\mathrm{~K}}\|\mathrm{Ta}-\mathrm{Tb}\|\right),
\end{aligned}
$$

$\forall \mathrm{a}, \mathrm{b} \in \mathrm{B}$ and $\forall \mathrm{t} \in[0,1] "$.

Lemma (1.8)[12]: "Let $M$ be a uniformly convex Banach space and its dual $M^{*}$ accomplishing the Kadec-Klee property.

Presume that $\left(a_{n}\right)$ bounded sequence in $M$ such as $\lim _{n \rightarrow \infty} \| t a_{n}+$ $(1-t) p_{1}-p_{2} \|$ exists $\forall t \in[0,1]$ and $p_{1}, p_{2} \in$ $W_{w}\left(a_{n}\right)$, thus $p_{1}=p_{2} "$.

\section{Main Results}

Let $\mathrm{B}$ be a nonempty closed convex subset of a Banach space $\mathrm{M}$ and $\left\{T_{j}, S_{j}, \forall j=1,2, \ldots, k\right\}$ be two families of total asymptotically quasi-nonexpansive self-maps. We define the iteration algorithm $\left(a_{n}\right)$ as follows:

$a_{1} \in B$

$a_{n+1}=\left(1-\alpha_{j n}\right) S_{j}^{n} a_{n}+\alpha_{j n} T_{j}^{n} b_{j n}$

$b_{j n}=\left(1-\alpha_{j n}\right) S_{j}^{n} a_{n}+\alpha_{j n} T_{j}^{n} b_{(j-1) n}$

$b_{(j-1) n}=\left(1-\alpha_{(j-1) n}\right) S_{j-1}^{n} a_{n}+\alpha_{(j-1) n} T_{j-1}^{n} b_{(j-2) n}$

$b_{2 n}=\left(1-\alpha_{2 n}\right) S_{2}^{n} a_{n}+\alpha_{2 n} T_{2}^{n} b_{1 n}$

$b_{1 n}=\left(1-\alpha_{1 n}\right) S_{1}^{n} a_{n}+\alpha_{1 n} T_{1}^{n} b_{0 n}$

Where $b_{0 n}=a_{n}$ and $\left(\alpha_{n}\right)_{n=1}^{\infty}$ are sequences in $[0,1]$.

Lemma (2.1): Let $B$ be a nonempty closed convex subset of a normed space $\mathrm{M}$ and $T_{j}, S_{j}, j=1,2, \ldots, k$ be two family of total asymptotically quasi-nonexpansive self-maps of B. Presume that $F\left(T_{j}, S_{j}\right) \neq \varnothing$ and the sequence $\left(a_{n}\right)$ be as shown in step (1).

Then:

i- There are sequences $\left(u_{n}\right)$ and $\left(v_{n}\right)$ in $[0, \infty)$ such as

$\sum_{n=1}^{\infty} u_{n}<\infty, \sum_{n=1}^{\infty} v_{n}<\infty$ and

$\left\|a_{n+1}-a^{*}\right\| \leq\left(1+u_{n}\right)^{j+1}\left\|a_{n}-a^{*}\right\|+v_{n}^{j+1}$,

$\forall a^{*} \in F\left(T_{j}, S_{j}\right)$ and $\forall n$.

ii- There exist constants $J_{1}, J_{2}>0$ such as

$\left\|a_{n+p}-a^{*}\right\| \leq J_{1}\left\|a_{n}-a^{*}\right\|+J_{2}, \forall a^{*} \in F\left(T_{j}, S_{j}\right)$

and $n, p=1,2, \ldots$..

If there is $Z>0$ such that $\psi\left(\lambda_{j}\right) \leq Z \lambda_{j}, j=1,2, \ldots, k$.

Proof: i- Let $a^{*} \in F, u_{n}=\max _{1 \leq j \leq k} f_{j n}$ and $v_{n}=\max _{1 \leq j \leq k} g_{j n}$.

Now, we have

$$
\begin{aligned}
\left\|b_{1 n}-a^{*}\right\|= & \left\|\left(1-\alpha_{1 n}\right) S_{1}^{n} a_{n}+\alpha_{1 n} T_{1}^{n} a_{n}-a^{*}\right\| \\
\leq & \left(1-\alpha_{1 n}\right)\left\|S_{1}^{n} a_{n}-a^{*}\right\|+\alpha_{1 n}\left\|T_{1}^{n} a_{n}-a^{*}\right\| \\
\leq & \left(1-\alpha_{1 n}\right)\left\{\left\|a_{n}-a^{*}\right\|+f_{1 n} \psi\left\|a_{n}-a^{*}\right\|+g_{1 n}\right\} \\
& \quad+\alpha_{1 n}\left\{\left\|a_{n}-a^{*}\right\|+f_{1 n} \psi\left\|a_{n}-a^{*}\right\|+g_{1 n}\right\} \\
\leq & \left(1-\alpha_{1 n}\right)\left(1+f_{1 n} Z\right)\left\|a_{n}-a^{*}\right\|+\left(1-\alpha_{1 n}\right) g_{1 n} \\
& \quad+\alpha_{1 n}\left(1+f_{1 n} Z\right)\left\|a_{n}-a^{*}\right\|+\alpha_{1 n} g_{1 n} \\
\leq & \left(1+f_{1 n} Z\right)\left\|a_{n}-a^{*}\right\|+g_{1 n} \\
\leq & \left(1+u_{n}\right)\left\|a_{n}-a^{*}\right\|+v_{n}
\end{aligned}
$$

Assume that $\left\|b_{j n}-a^{*}\right\| \leq\left(1+u_{n}\right)^{j}\left\|a_{n}-a^{*}\right\|+v_{n}^{j}$

Therefore,

$$
\begin{aligned}
\left\|b_{(j+1) n}-a^{*}\right\|= & \|\left(1-\alpha_{(j+1) n}\right) S_{j+1}^{n} a_{n}+\alpha_{(j+1) n} T_{j+1}^{n} b_{j} \\
& -a^{*} \|
\end{aligned}
$$




$$
\begin{aligned}
\leq & \left(1-\alpha_{(j+1) n}\right)\left\|S_{j+1}^{n} a_{n}-a^{*}\right\| \\
& +\alpha_{(j+1) n}\left\|T_{j+1}^{n} b_{j}-a^{*}\right\| \\
\leq & \left(1-\alpha_{(j+1) n}\right)\left(1+f_{(j+1) n} Z\right)\left\|a_{n}-a^{*}\right\| \\
& +\left(1-\alpha_{(j+1) n}\right) g_{(j+1) n} \\
& +\alpha_{(j+1) n}\left(1+f_{(j+1) n} Z\right)\left\|b_{j}-a^{*}\right\| \\
& +\alpha_{(j+1) n} g_{(j+1) n} \\
\leq & \left(1-\alpha_{(j+1) n}\right)\left(1+u_{n}\right)\left\|a_{n}-a^{*}\right\|+v_{n} \\
& +\alpha_{(j+1) n}\left(1+u_{n}\right)\left(1+u_{n}\right)^{j}\left\|a_{n}-a^{*}\right\| \\
& +\alpha_{(j+1) n}\left(1+u_{n}\right) v_{n}^{j} \\
\leq & \left(1+u_{n}\right)^{j+1}\left\|a_{n}-a^{*}\right\|+v_{n}^{j+1}
\end{aligned}
$$

Thus, by induction, we obtain

$$
\left\|b_{j n}-a^{*}\right\| \leq\left(1+u_{n}\right)^{j}\left\|a_{n}-a^{*}\right\|+v_{n}^{j}
$$

for all $j=1,2, \ldots, k$.

Now, by (2), we get

$$
\begin{aligned}
\left\|a_{n+1}-a^{*}\right\| \leq & \left(1-\alpha_{j n}\right)\left\|S_{j}^{n} a_{n}-a^{*}\right\|+\alpha_{j n}\left\|T_{j}^{n} b_{j n}-a^{*}\right\| \\
\leq & \left(1-\alpha_{j n}\right)\left(1+u_{n}\right)\left\|a_{n}-a^{*}\right\|+v_{n} \\
& +\alpha_{j n}\left(1+u_{n}\right)\left(1+u_{n}\right)^{j}\left\|a_{n}-a^{*}\right\| \\
& +\alpha_{j n}\left(1+u_{n}\right) v_{n}^{j} \\
\leq & \left(1+u_{n}\right)^{j+1}\left\|a_{n}-a^{*}\right\|+v_{n}^{j+1}
\end{aligned}
$$

ii- By using part (i), we get

$$
\begin{aligned}
\left\|a_{n+p}-a^{*}\right\| & \leq\left(1+u_{n+p-1}\right)^{j+1}\left\|a_{n+p-1}-a^{*}\right\|+v_{n+p-1}^{j+1} \\
& \leq e^{\left(1+u_{n+p-1}\right)^{j+1\left\|a_{n+p-1}-a^{*}\right\|}}+e^{v_{n+p-1}^{j+1}} \\
& \leq e^{(j+1) u_{n+p-1}\left\|a_{n+p-1}-a^{*}\right\|}+e^{(j+1) v_{n+p-1}} \\
& \leq e^{(j+1) \sum_{k=1}^{n+p-1} u_{k}}\left\|a_{n}-a^{*}\right\|+e^{(j+1) \sum_{k=1}^{n+p-1} v_{k}} \\
& \leq J_{1}\left\|a_{n}-a^{*}\right\|+J_{2} .
\end{aligned}
$$

Setting $J_{1}=e^{(j+1) \sum_{k=1}^{n+p-1} u_{k}}$ and $J_{2}=e^{(j+1) \sum_{k=1}^{n+p-1} v_{k}}$.

Lemma (2.2): Let $B$ be a nonempty closed convex subset of a normed space $\mathrm{M}$ and $T_{j}, S_{j}, j=1,2, \ldots, k$ be two families of total asymptotically quasi-nonexpansive self-maps of $\mathrm{B}$. Presume $F\left(T_{j}, S_{j}\right) \neq \varnothing$ and $\left(a_{n}\right)$ be as shown in step (1). Therefore, $\lim _{n \rightarrow \infty}\left\|a_{n}-a^{*}\right\|$ exists for all $a^{*} \in F\left(T_{i}, S_{i}\right)$.

Proof: By Lemma (2.1.i)

$$
\begin{aligned}
\left\|a_{n+1}-a^{*}\right\| & \leq\left(1+u_{n}\right)^{j+1}\left\|a_{n}-a^{*}\right\|+v_{n}^{j+1} \\
& \leq\left(1+u_{n}\right)\left\|a_{n}-a^{*}\right\|+v_{n}
\end{aligned}
$$

and $\sum_{n=1}^{\infty} u_{n}<\infty, \sum_{n=1}^{\infty} v_{n}<\infty$. So by Lemma (1.6.i), we get $\lim _{n \rightarrow \infty}\left\|a_{n}-a^{*}\right\|$ exists for all $a^{*} \in F\left(T_{j}, S_{j}\right)$.

Lemma (2.3): Let $B$ be a nonempty closed convex subset of a Banach space $M$ and $T_{j}, S_{j}, j=1,2, \ldots, k$ be two families of Lipschitzain and total asymptotically quasi-nonexpansive selfmaps of B. Let $\left(a_{n}\right)$ be as shown in step (1). Therefore, for all $a_{1}^{*}, a_{2}^{*} \in F\left(T_{j}, S_{j}\right)$, the limit $\lim _{n \rightarrow \infty}\left\|t a_{n}+(1-t) a_{1}^{*}-a_{2}^{*}\right\|$ exists for all $t \in[0,1]$.

If there is $Z>0$ such that $\psi\left(\lambda_{j}\right) \leq Z \lambda_{j}, j=1,2, \ldots, k$.

Proof: By using Lemma (1.6),we have $\lim _{n \rightarrow \infty}\left\|a_{n}-a^{*}\right\|$ exists $\forall a^{*} \in F\left(T_{j}, S_{j}\right)$ and $\left(a_{n}\right)$ is bounded. Let $\gamma_{n}(t)=\left\|t a_{n}+(1-t) a_{1}^{*}-a_{2}^{*}\right\|, \forall t \in[0,1]$.

Therefore, $\lim _{n \rightarrow \infty} a_{n}(0)=\left\|a_{1}^{*}-a_{2}^{*}\right\|$ and $\lim _{n \rightarrow \infty} a_{n}(1)=$ $\left\|a_{n}-a_{2}^{*}\right\|$ exist by Lemma (2.2).

Then, for $t \in[0,1]$ and for all $a \in B$, we define the map $R_{n}: B \rightarrow B$ by:

$$
\begin{aligned}
& b_{1 n}=\left(1-\alpha_{1 n}\right) S_{1}^{n} a_{n}+\alpha_{1 n} T_{1}^{n} b_{0 n} \\
& b_{2 n}=\left(1-\alpha_{2 n}\right) S_{2}^{n} a_{n}+\alpha_{2 n} T_{2}^{n} b_{1 n} \\
& \cdot \\
& \dot{b_{j n}}=\left(1-\alpha_{j n}\right) S_{j}^{n} a_{n}+\alpha_{i n} T_{j}^{n} b_{(j-1) n} \\
& R_{n} a=\left(1-\alpha_{j n}\right) S_{j}^{n} a_{n}+\alpha_{j n} T_{j}^{n} b_{j n}
\end{aligned}
$$

Now,

$$
\begin{aligned}
\left\|R_{n} a-R_{n} c\right\| \leq & \left(1-\alpha_{j n}\right)\left\|S_{j}^{n} a-S_{j}^{n} c\right\|+\alpha_{j n}\left\|T_{j}^{n} b_{j n}-T_{j}^{n} d_{j n}\right\| \\
\leq & \left(1-\alpha_{j n}\right)\left(1+f_{j n} Z\right)\|a-c\|+\left(1-\alpha_{j n}\right) g_{j n} \\
& +\alpha_{j n}\left(1+f_{j n} Z\right)\left\|b_{j n}-d_{j n}\right\|+\alpha_{j n} g_{j n} \\
\leq & \left(1+u_{n}\right)\|a-c\|+v_{n} \\
\leq & \left(1+f_{n} Z\right)\|a-b\|+g_{n}
\end{aligned}
$$

with $\sum_{n=1}^{\infty} u_{n}<\infty, \sum_{n=1}^{\infty} v_{n}<\infty$ and $s_{n}=1+u_{n}$, it follows that $s_{n} \rightarrow 1$ as $n \rightarrow \infty$.

Setting $W_{n, m}=R_{n+m-1} R_{n+m-1} \cdots R_{n}$ and $b_{n, m}=\| W_{n, m}\left(t a_{n}+(1-t) a_{1}^{*}-\left(t W_{n, m} a_{n}+(1-t) a_{1}^{*}\right) \|\right.$. Thus,

$$
\begin{aligned}
\left\|W_{n, m} a-W_{n, m} b\right\|= & \left\|\begin{array}{c}
R_{n+m-1} R_{n+m-2} \ldots R_{n}(a) \\
-R_{n+m-1} R_{n+m-2} \ldots R_{n}(b)
\end{array}\right\| \\
\leq & s_{n+m-1}\left\|\begin{array}{c}
R_{n+m-2} \ldots R_{n}(a) \\
-R_{n+m-2} \ldots R_{n}(b)
\end{array}\right\| \\
& +v_{n+m-1} \\
\leq & \prod_{j=n}^{n+m-1} s_{j}\|a-c\|+\sum_{j=n}^{n+m-1} v_{j} \\
= & A_{n}\|a-c\|+\sum_{j=n}^{n+m-1} v_{j}
\end{aligned}
$$

for all $a, c \in B$, where $A_{n}=\prod_{j=n}^{n+m-1} s_{j}, W_{n, m} a_{n}=$ $a_{n+m}$ and $a^{*}=a^{*}$ for all $a^{*} \in F\left(T_{i}, S_{i}\right)$.

Hence,

$$
\gamma_{n+m}(t)=\left\|t a_{n+m}+(1-t) a_{1}^{*}-a_{2}^{*}\right\|
$$

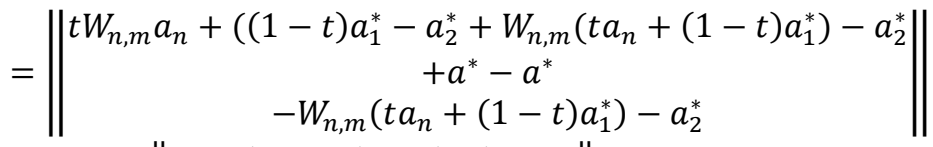$$
\leq b_{n, m}+\left\|W_{n, m}\left(t a_{n}+(1-t) a_{1}^{*}\right)-a_{2}^{*}\right\|
$$$$
\leq b_{n, m}+A_{n} \gamma_{n}(t)+\sum_{j=n}^{n+m-1} v_{j}
$$

By using Lemma (1.7), we have

$$
\begin{gathered}
b_{n, m} \leq K f^{-1}\left(\left\|a_{n}-a^{*}\right\|-\frac{1}{K}\left\|W_{n, m} a_{n}-W_{n, m} a^{*}\right\|\right) \\
\leq K f^{-1}\left(\left\|a_{n}-a^{*}\right\|-\frac{1}{K}\left(\left\|a_{n+m}-a^{*}\right\|\right.\right. \\
\left.-\left\|W_{n, m} a_{n}-a^{*}\right\|\right)
\end{gathered}
$$

and $\left(b_{n, m}\right)$ converges uniformly to zero. Since $\lim _{n \rightarrow \infty} A_{n}=$

1 and $\lim _{n \rightarrow \infty} v_{n}=0$, we get

$$
\begin{aligned}
\lim _{n \rightarrow \infty} \lim _{m \rightarrow \infty} \sup \gamma_{n+m} & \leq \lim _{n \rightarrow \infty} \lim _{m \rightarrow \infty} b_{n, m}+\lim _{n \rightarrow \infty} \inf \gamma_{n}(t) \\
& =\lim _{n \rightarrow \infty} \inf \gamma_{n}(t)
\end{aligned}
$$

Thus, $\lim _{n \rightarrow \infty} \gamma_{n}(t)$ exists for all $t \in[0,1]$. 
Theorem (2.4): Let $B$ be a nonempty closed convex subset of a uniformly convex Banach space $M, T_{j}, S_{j}, \forall j=1,2, \ldots, k$ be two families of Lipschiztain and total asymptotically quasinonexpansive self-maps of $\mathrm{B}$ and the sequence $\left(a_{n}\right)$ be as shown in step (1). If there is $Z>0$ such that $\psi\left(\left(\lambda_{j}\right) \leq Z \lambda_{j}, j=\right.$ $1,2, \ldots, k$. Then, $\lim _{n \rightarrow \infty}\left\|T_{j}^{n} a_{n}-a_{n}\right\|=0=\lim _{n \rightarrow \infty}\left\|S_{j}^{n} a_{n}-a_{n}\right\|, \forall j$. Proof: By Lemma (2.2), $\lim _{n \rightarrow \infty}\left\|a_{n}-a^{*}\right\|$ exists. Assume that

$$
\lim _{n \rightarrow \infty}\left\|a_{n}-a^{*}\right\|=e, \quad \forall e \geq 0 .
$$

If $\mathrm{e}=0$, the proof is straight forward.

Now, suppose $e>0$. We get

and

$$
a_{n+1}=\left(1-\alpha_{j n}\right) S_{j}^{n} a_{n}+\alpha_{j n} T_{j}^{n} b_{j n}
$$

$$
\begin{aligned}
e=\left\|a_{n+1}-a^{*}\right\| & =\|\left(1-\alpha_{j n}\right)\left(S_{j}^{n} a_{n}-a^{*}\right)+\alpha_{j n}\left(T_{j}^{n} b_{j n}\right. \\
& \left.-a^{*}\right) \| .
\end{aligned}
$$

Since $\left\|S_{j}^{n} a_{n}-a^{*}\right\| \leq K\left\|a_{n}-a^{*}\right\| \leq e$

and $\left\|T_{j}^{n} b_{j n}-a^{*}\right\| \leq K\left\|b_{j n}-a^{*}\right\| \leq K\left\{\left(1+u_{n}\right)^{j} \| a_{n}-\right.$

$$
\left.a^{*} \|+v_{n}^{j}\right\} \leq e .
$$

Therefore, by Lemma (1.5), we get

$$
\lim _{n \rightarrow \infty}\left\|S_{j}^{n} a_{n}-T_{j}^{n} b_{j n}\right\|=0
$$

Next,

$$
\begin{aligned}
\left\|b_{j n}-a_{n}\right\| \leq & \left(1-\alpha_{j n}\right)\left\|S_{j}^{n} a_{n}-a_{n}\right\|+\alpha_{j n}\left\|T_{j}^{n} b_{(j-1) n}-a_{n}\right\| \\
\leq & \left(1-\alpha_{j n}\right)\left(1+f_{j n} Z\right)\left\|a_{n}-a_{n}\right\|\left(1-\alpha_{j n}\right) g_{j n} \\
& \quad+\alpha_{j n}\left(1+f_{j n} Z\right)\left\|b_{(j-1) n}-a_{n}\right\|+\alpha_{j n} g_{j n} \\
\leq & g_{j n}+\alpha_{j n}\left(1-\alpha_{(j-1) n}\right)\left(1+f_{j n} Z\right) \\
& \left\|S_{j-1}^{n} a_{n}-a_{n}\right\|+\alpha_{j n} \alpha_{(j-1) n}\left(1+f_{j n} Z\right) \\
& \left\|T_{j-1}^{n} b_{(j-2) n}-a_{n}\right\| \\
\leq & g_{j n}+\alpha_{j n}\left(1-\alpha_{(j-1) n}\right)\left(1+f_{j n} Z\right) g_{j n} \\
& +\alpha_{j n} \alpha_{(j-1) n}\left(1+f_{j n} Z\right)(1 \\
& \left.+f_{(j-1) n} Z\right)\left\|b_{(j-2) n}-a_{n}\right\| \\
& +\alpha_{j n} \alpha_{(j-1) n}\left(1+f_{j n} Z\right) g_{(j-1) n}
\end{aligned}
$$

$$
\text { . }
$$

$<\infty$

Since $Z>0, \sum_{n=1}^{\infty} f_{j n}<\infty$ and $\sum_{n=1}^{\infty} g_{j n}<\infty$, hence

$\lim _{n \rightarrow \infty}\left\|b_{j n}-a_{n}\right\|=0$.

Then,

$$
\begin{aligned}
\left\|S_{j}^{n} a_{n}-a_{n}\right\| & \leq\left\|S_{j}^{n} a_{n}-T_{j}^{n} b_{j n}\right\|+\left\|T_{j}^{n} b_{j n}-a_{n}\right\| \\
& \leq\left\|S_{j}^{n} a_{n}-T_{j}^{n} b_{j n}\right\|+K\left\|b_{j n}-a_{n}\right\| \rightarrow 0 \\
\text { as } n \rightarrow \infty . &
\end{aligned}
$$

And

$\left\|T_{j}^{n} a_{n}-a_{n}\right\| \leq\left\|T_{j}^{n} a_{n}-S_{j}^{n} a_{n}\right\|+\left\|S_{j}^{n} a_{n}-a_{n}\right\| \rightarrow 0$

Theorem (2.5): Let B be a nonempty closed convex subset of a Banach space and $T_{j}, S_{j}, j=1,2, \ldots, k$ be two families of total asymptotically quasi-nonexpansive self-maps of B. Presume that $F\left(T_{j}, S_{j}\right) \neq \varnothing$ and $\left(a_{n}\right)$ be as shown in step (1)is strong convergence to a common fixed point of $T_{j}$ and $S_{j}$ iff $\lim _{n \rightarrow \infty} \inf d\left(a_{n}, F\right)=0$, where $d(a, F)=i n f_{a^{*} \in F}\left\|a-a^{*}\right\|$.

Proof: To show $\lim _{n \rightarrow \infty} \inf d\left(a_{n}, F\right)=0$ implies that $\left(a_{n}\right)$ is strong convergence to a common fixed point of $T_{j}, S_{j}, j=$ $1,2, \ldots, k$, since by $(2)$

www.astesj.com

$$
\begin{aligned}
d\left(a_{n+1}, F\right) & \leq\left(1+u_{n}\right)^{j+1} d\left(a_{n}, F\right)+v_{n}^{j+1} \\
& \leq\left(1+u_{n}\right) d\left(a_{n}, F\right)+v_{n}
\end{aligned}
$$

By Lemma (1.6), we get $\lim _{n \rightarrow \infty} a_{n}$ exists and $\lim _{n \rightarrow \infty}$ inf $a_{n}=0$.

Hence, $\lim _{n \rightarrow \infty} a_{n}=0$.

Next, to prove the sufficiency, firstly show that $\left(a_{n}\right)$ Cauchy sequence. By using Lemma (2.1.ii), we get

$$
\left\|a_{n+p}-a^{*}\right\| \leq J_{1}\left\|a_{n}-a^{*}\right\|+J_{2}
$$

$\forall a^{*} \in F\left(T_{j}, S_{j}\right), n=p=1,2, \ldots$

Since $\lim _{n \rightarrow \infty} a_{n}=0, \forall \epsilon>0, \exists N$ such that

$$
d\left(a_{n}, F\right) \leq \frac{\epsilon}{3 J_{1}}-\frac{J_{2}}{J_{1}}, \quad \forall n \geq N
$$

therefore, there is $l \in F\left(T_{i}, S_{i}\right)$ such that

$$
\left\|a_{N}-l\right\| \leq \frac{\epsilon}{2 J_{1}}-\frac{J_{2}}{J_{1}}
$$

From (3) and (4), $\forall n \geq N$, we get

$$
\begin{aligned}
\left\|a_{n+p}-a_{n}\right\| & \leq\left\|a_{n+p}-l\right\|+\left\|a_{n}-l\right\| \\
& \leq J_{1}\left\|a_{N}-l\right\|+J_{2}+J_{1}\left\|a_{N}-l\right\|+J_{2} \\
& \leq J_{1} \frac{\epsilon}{2 J_{1}}-J_{2}+J_{2}+J_{1} \frac{\epsilon}{2 J_{1}}-J_{2}+J_{2} \\
& =\epsilon
\end{aligned}
$$

Then, $\left(a_{n}\right)$ is a Cauchy sequence and converges to $c \in M$. Lastly, to show that $c \in F\left(T_{j}, S_{j}\right)$, for any $\epsilon^{*}>0$, there is $N_{1}$ such that

$$
\left\|a_{n}-c\right\| \leq \frac{\epsilon^{*}}{2\left(2+f_{j} Z\right)}-\frac{3 g_{j}}{\left(2+f_{j} Z\right)}, \forall n \geq N_{1}
$$

Since $\lim _{n \rightarrow \infty} a_{n}=0$ implies that $N_{2} \geq N_{1}$ such that $d\left(a_{n}, F\right) \leq$ $\frac{\epsilon^{*} n \rightarrow \infty}{3\left(4+3 f_{j} z\right)}, \forall n \geq N_{2}$.

Thus, $\exists l_{1} \in F\left(T_{j}, S_{j}\right)$ such that

$$
\left\|a_{N}-l_{1}\right\| \leq \frac{\epsilon^{*}}{2\left(4+3 f_{j} z\right)}
$$

From (5) and (6) for any $T_{j}, \forall j=1,2, \ldots, k$, we obtain

$$
\begin{aligned}
& \left\|T_{j} c-c\right\| \leq\|j c-l\|+2\left\|T_{j} a_{N_{2}}-l_{1}\right\|+\left\|a_{N_{2}}-l\right\| \\
& +\left\|a_{N_{2}}-c\right\| \\
& \leq\left\|c-l_{1}\right\|+f_{j} \psi\left\|p-l_{1}\right\|+g_{j}+2\left\|a_{N_{2}}-l_{1}\right\| \\
& +2 f_{j} \psi\left\|a_{N_{2}}-l\right\|+2 g_{j}+\left\|a_{N_{2}}-l_{1}\right\| \\
& +\left\|a_{N_{2}}-c\right\| \\
& \leq\left(1+f_{j} Z\right)\left\|c-l_{1}\right\|+2\left(1+f_{j} Z\right)\left\|a_{N_{2}}-l_{1}\right\|+3 g_{j} \\
& +\left\|a_{N_{2}}-l_{1}\right\|+\left\|a_{N_{2}}-c\right\| \\
& \leq\left(1+f_{j} Z\right)\left\|a_{N_{2}}-c\right\|+\left(1+f_{j} Z\right)\left\|a_{N_{2}}-l_{1}\right\| \\
& +2\left(1+f_{j} Z\right)\left\|a_{N_{2}}-l_{1}\right\|+3 g_{j}+\left\|a_{N_{2}}-l_{1}\right\| \\
& +\left\|a_{N_{2}}-c\right\| \\
& \leq\left(2+f_{j} Z\right)\left\|a_{N_{2}}-c\right\|+\left(4+3 f_{j} Z\right)\left\|a_{N_{2}}-l_{1}\right\| \\
& +3 g_{j} \\
& \leq\left(2+f_{j} Z\right) \frac{\epsilon^{*}}{2\left(2+f_{j} Z\right)}-3 g_{j}+3 g_{j} \\
& +\left(4+3 f_{j} Z\right) \frac{\epsilon^{*}}{2\left(4+3 f_{j} Z\right)} \\
& =\epsilon^{*}
\end{aligned}
$$

Therefore, $\left\|T_{j} c-c\right\|=0 \forall i$ which means $T_{j} c=c, \forall j=$ $1,2, \ldots, k$.

By using the same above argument we can prove $\left\|S_{j} c-c\right\|=$ $0, \forall j$. Thus $c \in F\left(T_{j}, S_{j}\right)$. 
Theorem (2.6): Let B be a nonempty closed convex subset of a uniformly convex Banach space and $T_{j}, S_{j}, j=1,2, \ldots, k$ be two families of Lipschitzain and total asymptotically quasinonexpansive self-maps of B. If $\mathrm{M}$ accomplishes Opial 's condition and the maps $I-T_{j}$ and $I-S_{j}, j=1,2, \ldots, k$ are demiclosed to zero, therefore $\left(a_{n}\right)$ be as shown in step (1) is weak convergence to a common fixed point of $T_{j}$ and $S_{j}, j=$ $1,2, \ldots, k$.

Proof: Let $a^{*} \in F\left(T_{j}, S_{j}\right)$. By Lemma (2.2), $\lim _{n \rightarrow \infty}\left\|a_{n}-a^{*}\right\|$

exists.

By Theorem (2.4), we have

$$
\lim _{n \rightarrow \infty}\left\|T_{j}^{n} a_{n}-a_{n}\right\|=0=\lim _{n \rightarrow \infty}\left\|S_{j}^{n} a_{n}-a_{n}\right\|
$$

for $\mathrm{j}=1,2, \ldots, \mathrm{k}$. Since by the supposition the maps $I-$ $T_{j}$ and $I-S_{j}, \forall j=1,2, \ldots, k$ are demiclosed to zero, therefore $T_{j} a^{*}=a^{*}$ and $S_{j} a^{*}=a^{*}$, that means $a^{*} \in F\left(T_{j}, S_{j}\right)$.

Next, to prove $\left(a_{n}\right)$ converges weakly to $a^{*}$. Assume there is other subsequence $\left(a_{n i}\right)$ of $\left(a_{n}\right)$ is weak convergence to $b^{*} \in$ $F\left(T_{j}, S_{j}\right)$ and $a^{*} \neq b^{*}$. By using the same argument as above we can show that $b^{*} \in F\left(T_{j}, S_{j}\right)$.

Now, to prove the uniqueness, assume $a^{*} \neq b^{*}$. Therefore, by using Opial 's condition, we obtain:

$$
\begin{aligned}
\lim _{n \rightarrow \infty}\left\|a_{n}-a^{*}\right\| & =\lim _{n j \rightarrow \infty}\left\|a_{n j}-a^{*}\right\| \\
& <\lim _{n j \rightarrow \infty}\left\|a_{n j}-b^{*}\right\| \\
& =\lim _{n i \rightarrow \infty}\left\|a_{n i}-b^{*}\right\| \\
& <\lim _{n \rightarrow \infty}\left\|a_{n i}-a^{*}\right\| \\
& =\lim _{n \rightarrow \infty}\left\|a_{n}-a^{*}\right\|
\end{aligned}
$$

This is contradiction, therefore $a^{*} \neq b^{*}$. Hence, $\left(a_{n}\right)$ is weak convergence to $a^{*}$.

Theorem (2.7): Let B be a nonempty closed convex subset of a uniformly convex Banach space and $T_{j}, S_{j}, j=1,2, \ldots, k$ be two families of Lipschitzain and total asymptotically quasi-

nonexpansive self-maps of B. If the dual space $M^{*}$ of $M$ has the Kadec-klee property and the maps $I-T_{j}, I-S_{j}, j=1,2, \ldots, k$ are demi-closed to zero, therefore, $\left(a_{n}\right)$ be as shown in step (1) is weak convergence to a common fixed point of $T_{j}$ and $S_{j}$.

Proof: As showed by Lemma (2.2), that $\lim _{n \rightarrow \infty}\left\|a_{n}-a^{*}\right\|$ exists.

Since $\left(a_{n}\right)$ is bounded in B and $M$ is reflexive. Therefore, there exists a subsequence $\left(a_{n i}\right)$ of $\left(a_{n}\right)$ which is weak convergence to a point $a^{*} \in B$. By Theorem (2.4)

$\forall \mathrm{j}=1,2, \ldots, \mathrm{k}$.

$$
\lim _{n \rightarrow \infty}\left\|T_{j}^{n} a_{n}-a_{n}\right\|=0=\lim _{n \rightarrow \infty}\left\|S_{j}^{n} a_{n}-a_{n}\right\|
$$

Since by the supposition the maps $I-T_{j}$ and $I-S_{j}, \forall j=$ $1,2, \ldots, k$ are demi-closed to zero. Thus, $a^{*} \in F\left(T_{j}, S_{j}\right)$. Now, to prove $\left(a_{n}\right)$ is weak convergence to a point $a^{*}$. Presume that $\left(a_{n k}\right)$ is other subsequence of $\left(a_{n}\right)$ that is weak convergence to a point $b^{*} \in F\left(T_{j}, S_{j}\right)$. By using the same argument as above, we obtain $b^{*} \in F\left(T_{j}, S_{j}\right)$.

Therefore, by Lemma (2.3) $\lim _{n \rightarrow \infty}\left\|t a_{n}+(1-t) a^{*}-b^{*}\right\|$ exists for all $t \in[0,1]$.

By Lemma (1.8) $a^{*}=b^{*}$. As a result, the sequence $\left(a_{n}\right)$ is weak convergence to the point $a^{*} \in F\left(T_{j}, S_{j}\right)$.
The following corollaries are special cases

Corollary (2.8): Let $B$ be a nonempty closed convex subset of a Banach space and $T_{j}, S_{j}, j=1,2, \ldots, k$ be two families of total asymptotically nonexpansive self-maps of B. Presume that $F\left(T_{j}, S_{j}\right) \neq \varnothing$ and $\sum_{n=1}^{\infty} f_{n}<\infty, \sum_{n=1}^{\infty} g_{n}<\infty$.

Presume that $\left(a_{n}\right)$ be as shown in step (1) is strong convergence to a a common fixed point of $T_{j}$ and $S_{j}$ iff $\lim _{n \rightarrow \infty} \inf d\left(a_{n}, F\right)=0$, where $d(a, F)=i n f_{a^{*} \in F}\left\|a-a^{*}\right\|$. Corollary (2.9): Let $T_{j}, S_{j}, B, f_{j}$ and $g_{j}, j=1,2, \ldots, k$ be as in corollary (2.8). Therefore $\left(a_{n}\right)$ be as shown in step (1) is strong convergence to $a^{*} \in F\left(T_{j}, S_{j}\right)$ iff $\left(a_{n i}\right)$ of $\left(a_{n}\right)$ that converges to $a^{*}$.

Corollary (2.10): Let $B$ be a nonempty closed convex subset of a uniformly convex Banach space and $T_{j}, S_{j}, \forall j=1,2, \ldots, k$ be two families of Lipschitzain and total asymptotically nonexpansive self-maps of B. If the dual space $M^{*}$ of $M$ has the Kadec-klee property and the maps $I-T_{j}$ and $I-S_{j}, \forall j=$ $1,2, \ldots, k$ are demi-closed to zero, therefore $\left(a_{n}\right)$ be as shown in step (1) is weak convergence to a common fixed point of $T_{i}$ and $S_{i}$.

Corollary (2.11): Let $B$ be a nonempty closed convex subset of a uniformly convex Banach space and $T_{j}, S_{j}, \forall j=1,2, \ldots, k$ be two families of total asymptotically nonexpansive self-maps of B. If $\mathrm{M}$ accomplishes Opial 's condition and the maps $I-$ $T_{j}$ and $I-S_{j}, \forall j=1,2, \ldots, k$ are demi-closed to zero, therefore, $\left(a_{n}\right)$ be as shown in step (1) is weak convergence to a common fixed point of $T_{j}, S_{j}, \forall j=1,2, \ldots, k$.

Corollary (2.12): Let $B$ be a nonempty closed convex subset of a Banach space and $T_{j}, S_{j}, j=1,2, \ldots, k$ be two families of asymptotically quasi-nonexpansive self-maps of B. Presume that $F\left(T_{j}, S_{j}\right) \neq \varnothing$ and $\sum_{n=1}^{\infty} f_{n}<\infty$. Presume that $\left(a_{n}\right)$ be as shown in step (1) is strong convergence to a common fixed point of $T_{j}$ and $S_{j}$ iff $\lim _{n \rightarrow \infty} \inf d\left(a_{n}, F\right)=0$, where $d(a, F)=$ inf $f_{a^{*} \in F}\left\|a-a^{*}\right\|$.

Corollary (2.13): Let $B$ be a nonempty closed convex subset of a uniformly convex Banach space and $T_{j}, S_{j}, \forall j=1,2, \ldots, k$ be two families of Lipschitzain and asymptotically quasinonexpansive self-maps of B. If the dual space $M^{*}$ of $M$ has the Kadec-klee property and the maps $I-T_{j}$ and $I-S_{j}, \forall j=$ $1,2, \ldots, k$ are demi-closed to zero, therefore, $\left(a_{n}\right)$ be as shown in step (1) is weak convergence to a common fixed point of $T_{j}$ and $S_{j}$.

Corollary (2.14): Let $B$ be a nonempty closed convex subset of a uniformly convex Banach space and $T_{j}, S_{j}, \forall j=1,2, \ldots, k$ be two families of Lipschitzain and asymptotically quasinonexpansive selfmaps of $\mathrm{B}$. If $\mathrm{M}$ accomplishes Opial 's condition and the maps $I-T_{j}$ and $I-S_{j}, \forall j=1,2, \ldots, k$ are demi-closed to zero, therefore, $\left(a_{n}\right)$ be as shown in step $(1)$ is weak convergenve to a common fixed point of $T_{j}$ and $S_{j}, \forall j=$ $1,2, \ldots, k$.

\section{Numerical Example}

We illustrate our results by the following 
Example (3.1): Let $T_{j}, S_{j}: R \rightarrow R, \forall j=1,2, \ldots, k$ be two maps such that $T_{j} a=\frac{2 a}{3^{j}}$ and $S_{j} a=\frac{46 a}{2^{j}}, \forall a \in R$. Choose $\alpha_{j n}=$ $\frac{n}{4(n+1)} \forall n$ with initial value $a_{1}=15$. Let $\left(a_{n}\right)$ be the sequence. According to Table 1 and Figure 1, it eases to sight that $\left(a_{n}\right)$ converges to the fixed point $a^{*}=0$.

Table 1: Numerical results corresponding to $a_{1}=15$ for 36 steps.

\begin{tabular}{|c|c|c|c|}
\hline $\mathbf{n}$ & Iteration (1) & $\mathbf{n}$ & Iteration (1) \\
\hline $\mathbf{1}$ & 15.0000 & $\mathbf{1 3}$ & 0.0184 \\
\hline $\mathbf{2}$ & 9.4401 & $\mathbf{1 4}$ & 0.0102 \\
\hline $\mathbf{3}$ & 5.6594 & $\mathbf{1 5}$ & 0.0056 \\
\hline $\mathbf{4}$ & 3.3084 & $\mathbf{1 6}$ & 0.0031 \\
\hline $\mathbf{5}$ & 1.9044 & $\mathbf{1 7}$ & 0.0017 \\
\hline $\mathbf{6}$ & 1.0849 & $\mathbf{1 8}$ & 0.0009 \\
\hline $\mathbf{7}$ & 0.6134 & $\mathbf{1 9}$ & 0.0005 \\
\hline $\mathbf{8}$ & 0.3448 & $\mathbf{2 0}$ & 0.0003 \\
\hline $\mathbf{9}$ & 0.1930 & $\mathbf{2 1}$ & 0.0002 \\
\hline $\mathbf{1 0}$ & 0.1076 & $\mathbf{2 2}$ & 0.0001 \\
\hline $\mathbf{1 1}$ & 0.0599 & $\mathbf{2 3}$ & 0.0000 \\
\hline $\mathbf{1 2}$ & 0.0332 & $\mathbf{2 4}$ & 0.0000 \\
\hline
\end{tabular}

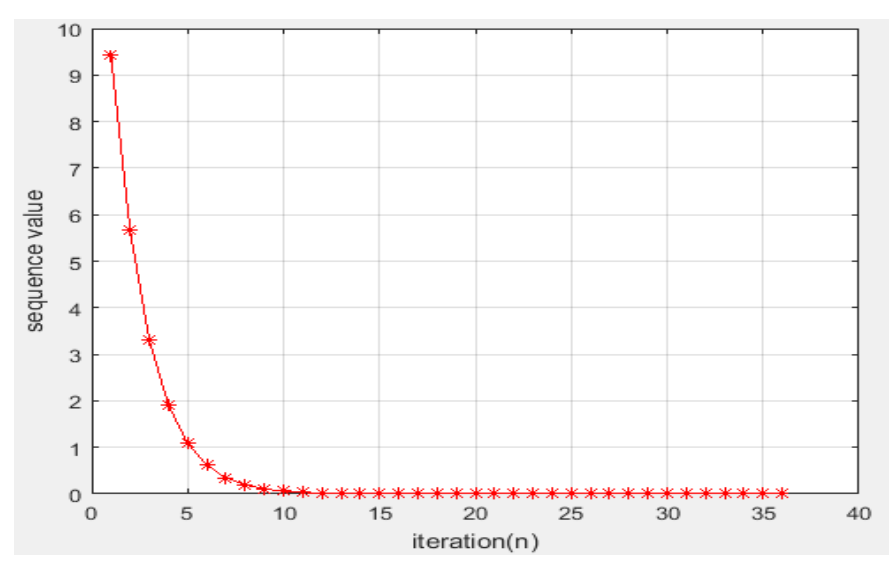

Figure. 1. Convergence behavior corresponding to $a_{1}=15$ for 36 steps.

\section{Conclusion}

We study the strongly and weakly convergence of new type of finite-step iteration processes under total asymptotically quasinonexpansive maps, see Theorems (2.4)-(2.6). Our results are generalizing and unifying the results of others who have been referred to in the references.

\section{Open Problem}

Recently, S.S. Abed has been defined as the following type of generalizations of total asymptotically quasi-nonexpansive[16]: Let $\mathcal{A}$ be a subset of real Banach space a set-valued map $G: \mathcal{A}$ $\rightarrow 2^{\mathcal{A}}$ is called the general asymptotic set-valued if for each $x \in$ $\mathcal{A}$ there exists null non- negative real sequences $\left\{\mathrm{a}_{\mathrm{n}}\right\}$ and $\left\{\mathrm{b}_{\mathrm{n}}\right\}$ such that

$$
\mathfrak{D}\left(G^{n} x, G^{n} \mathrm{y}\right) \leq\|x-w\|+a_{n} \mu(\|x-w\|)+b_{n}
$$

for any $y \in \mathcal{A}, w \in G^{n} \mathrm{y}$ and $\mu: R^{+} \rightarrow R^{+}$with $\mu(0)=0$.

One can study convergence theorems in (1) and in [17, theorem (11)] for families of general asymptotic set-valued maps. As well as possible to demonstrate new results in the case of other spaces as a modular space [18].

\section{Acknowledgment}

The authors wish to express his thanks to the referees for their helpful advice.

\section{References}

[1] S.S.Abed, Z.M Mohamed Hasan "Convergence theorems of a finite-step iteration algorithm under two finite families of total asymptotically quasinonexpansive maps" IEEE Xplore Digital Library Internationl Conference on Advanced Science and Engineering (ICOASE), , 2018.

[2] U K.Goebel, W. A. Kirk, "Fixed point theorem for asymptotically nonexpansive mappings" Proc. Amer. Math. Soc. 35, 171-174, 1972.

[3] W. V. Petryshyn, T. E. Williamson, "Strong and weak convergence of the sequence of successive approximations for quasi-nonexpansive mappings", J. Math. Anal. Appl. 43, 459-497, 1973.

[4] YA. I. Alber, C. E. Chidume, H. Zegeye, " Approximating fixed points of total asymptotically nonexpansive mappings" Fixed point theory Appl. 2006, article ID 10673.

[5] G. S. Saluja, "Convergence to common fixed points for generalized asymptotically quasi-nonexpansive mappings" Bull. Soc. Math. 4, 69-79, 2014.

[6] G. S. Saluja, "Strong convergence theorems for two finite families of generalized asymptotically quasi-nonexpansive mappings with application" Math. Moravica, 22(1), 1-14, 2018.

[7] S.S.Abed, Z.M Mohamed Hasan,."Common fixed point of a finite-step iteration algorithm under total asymptotically quasi-nonexpansive maps" is accepted in Baghdad Science Journal.

[8] J. B. Diaz, F. B. Metcalf, "On the structure of the set of subsequential limit points of successive approximation" Bull. Amer. Math. Soc., 73, 516-519, 1967.

[9] D. R, Sahu, D. ORegan, R. P. Agarwal ,"Fixed point theorey for Lipschitzain-type mappings with applications, Topological fixed point theory and its applications" doi:10.1007/978-387-75818-3-1.

[10] Q. H. Liu, "Iterative sequences for asymptotically quasi-nonexpansive mappings" J. Math. Anal. Appl. 259(1), 1-7, 2001

[11] A. Abkar, M. Shekarbaigi,"A novel iteration algorithm applied to totally asymptotically nonexpansive mappings in CAT(0) spaces" Mathematices 5,14, 2017, doi:10.3390/math5010014.

[12] F.E.Browder," Semicontractive and semiaccretive nonlinear mappings in Banach spaces" Bull. Amer. Math. Soc. 74, 660-665, 1968.

[13] A.Sharma, M.Imdad, "Approximating fixed points of generalized nonexpansive mappings Via faster iteration schemes" Fixed point theory, 4(4), 605-623, 2014.

[14] C. E. Chidume, E.U. Ofoedu, "Approximation of common fixed points for finite families of total asymptotically nonexpansive mappings" J. Math. Anal. Appl. 333, 128-141, 2007,doi:10.1016/j.jmaa.2006.09.023.

[15] J.G. Falset, W. Kaczor, T. Kuczumow, S. Reich, " Weak convergence theorem for asymptotically nonexpansive mappings and semigroups", Nonlinear Anal. 43, 377- 401, 2001.

[16] S.S.Abed, "Approximating fixed points of the general set -valued asymptotically mappings " to appear.

[17] S.S.Abed, Z.M Mohamed Hasan "Convergence comparison of two schemes for common fixed points with an application" accepted in Ibn Al Haitham journal for pure and applied sciences.

[18] S.S.Abed, K. E. Abdul Sada, " Common fixed points in modular spaces", IHSCICONF 2017, Ibn Al Haitham journal for pure and applied sciences. 